2000

\title{
Coherent structure analysis of spatiotemporal chaos
}

PeterJung

Jichang Wang

Renate Wackerbauer

Kenneth Showalter

Follow this and additional works at: https://researchrepository.wvu.edu/faculty_publications

\section{Digital Commons Citation}

Jung, Peter; Wang, Jichang; Wackerbauer, Renate; and Showalter, Kenneth, "Coherent structure analysis of spatiotemporal chaos" (2000). Faculty Scholarship. 84.

https://researchrepository.wvu.edu/faculty_publications/84 


\title{
Coherent structure analysis of spatiotemporal chaos
}

\author{
Peter Jung \\ Department of Physics and Astronomy, Ohio University, Athens, Ohio 45701 \\ Jichang Wang, Renate Wackerbauer, and Kenneth Showalter \\ Department of Chemistry, West Virginia University, Morgantown, West Virginia 26506-6045
}

(Received 18 May 1999)

\begin{abstract}
We introduce a measure to quantify spatiotemporal turbulence in extended systems. It is based on the statistical analysis of a coherent structure decomposition of the evolving system. Applied to a cellular excitable medium and a reaction-diffusion model describing the oxidation of $\mathrm{CO}$ on $\mathrm{Pt}(100)$, it reveals power-law scaling of the size distribution of coherent space-time structures for the state of spiral turbulence. The coherent structure decomposition is also used to define an entropy measure, which sharply increases in these systems at the transition to turbulence.
\end{abstract}

PACS number(s): 82.40.Bj, 05.45.-a, 05.65.+b, 47.54.+r

Low-dimensional chaos is now well understood and measures for its characterization are highly developed; however, the characterization of spatiotemporal chaos remains an important challenge. Several avenues have been explored in recent years to establish measures of turbulent behavior in distributed systems, such as extensive scaling of Liapunov exponents, fractal dimensions, and spatial and temporal correlation functions (for a review, see Ref. [1]). Hildebrand et al. [2] have described spiral turbulence in terms of defect densities, i.e., the density of centers of rotation, where the number of defects was found to fluctuate around a constant mean value. Principal orthogonal decomposition in space and time [Karhunen-Loeve decomposition] has also been utilized to characterize spatiotemporal chaos [3]. The number of orthogonal components necessary to reconstruct the essential dynamical features of a spatiotemporal chaotic system has been found to scale extensively with the system size, and extensive scaling has been revealed for spiral defect chaos in a convection system [4].

In this paper, we describe a method for characterizing spatiotemporal dynamics that is based on a statistical analysis of the associated spatiotemporal behavior [5]. We create a decomposition of the space-time matrix in terms of clusters of correlated events in space and time. The birth and death of a space-time cluster are directly related to particular dynamical events, and the cluster is therefore linked to the physical mechanism underlying the spatiotemporal behavior. Furthermore, coherent cluster decomposition allows the reconstruction of specific features of the spatiotemporal dynamics, such as cluster-size statistics and associated scaling laws. We have applied this method to a cellular excitable medium and a reaction-diffusion model describing the oxidation of $\mathrm{CO}$ on $\mathrm{Pt}(100)$. We find power-law scaling of the cluster-size distribution in each model for the state of spiral turbulence as well as an increase in spatiotemporal entropy at the onset of this behavior.

Coherent cluster analysis. We consider the spatiotemporal evolution of a two-dimensional extended dynamical system. In the first step, we stack a temporal sequence of $N_{t}$ snapshots of the system, taken at times $t_{n}=n \Delta t$, to obtain a space-time cube that carries all of the spatiotemporal infor- mation within the time interval $N_{t} \Delta t$. In the second step, we perform a binary reduction of the data by imposing a threshold to distinguish between active and inactive sites. Active sites that are connected as neighbors (in the time-forward direction) form objects which we term coherent space-time clusters [6]. In the third step, a decomposition of the coherent clusters is carried out. If two clusters collide and merge into a single cluster, the younger contributing cluster is terminated at the collision point in order to avoid counting two structures from uncorrelated sources as one coherent structure. The size $s$ of each of the coherent clusters, i.e., the number of active elements constituting a cluster, is characterized by the cluster-size distribution function $p_{s}$.

Spiral turbulence in a cellular excitable medium. We consider a square array of excitable three-state cells $e_{i j}$ with lattice spacing $a$. The voltage controling the state of the cell $e_{i j}$ is denoted by $\mathrm{v}_{i j}$. When the voltage $\mathrm{v}_{i j}$ crosses the threshold $b$, the cell $e_{i j}$ fires, changing from the quiescent to the excited state. Immediately after firing, the cell becomes refractory, where it is not excitable during a refractory period with zero voltage. In the quiescent state, each cell is dissipative, with an exponential decay (with decay constant $\gamma$ ) of the voltages $\mathrm{v}_{i j}$,

$$
\mathrm{v}_{i j}(t+\Delta t)=\mathrm{v}_{i j}(t) \exp (-\gamma \Delta t)+\sum_{k l} G(i, j ; k, l) \rho(k, l, t) .
$$

The additional term on the right-hand side of this equation describes the impact of pulses emitted by other cells $e_{k l}$ firing at time $t$. The indicator function $\rho(k, l, t)$ is unity for cells that are firing at time $t$ and zero otherwise. It is determined by the voltages $\mathrm{v}_{k l}$ at time $t$, i.e., $\rho(k, l, t)=\Theta\left(\mathrm{v}_{k l}(t)-b\right)$ with the Heavyside function $\Theta(\cdot)$. The Green's function $G(i, j ; k, l)$ is defined as

$$
G(i, j ; k, l)=K \exp \left(-\lambda \frac{r_{i j, k l}^{2}}{a^{2}}\right),
$$

with $r_{i j, k l}$ being the distance between the emitting and the receiving cell and $\lambda$ describing the interaction range between 
the cells. While under most conditions, this model reproduces the typical patterns of excitable media (such as the FitzHugh-Nagumo model [7]), it exhibits spatiotemporal disorder in the form of spiral turbulence at weak damping $\gamma$ and weak coupling $K$. The interaction between firing and quiescent cells results in the nucleation of daughter waves, which collide with parent waves to give rise to spatiotemporal disorder. A typical turbulent pattern for $K$ close to the propagation threshold $K_{0}$ is shown in Fig. 1(a). Far from the propagation threshold, the pattern is characterized by the coexistence of many spiral waves [Fig. 1(b)], similar to the convective spiral turbulence observed by $\mathrm{Hu}$ et al. [8]. Applying the coherent cluster algorithm to these chaotic patterns reveals the power-law distribution of cluster sizes shown in Fig. 1(c). The range and slope of the power law depends on the value of the coupling constant $K$. Close to the propagation threshold $K_{0}$, the patterns exhibit the most irregular shapes and we find a power law with an exponent of approximately 2.0. As we increase the coupling, the patterns become more ordered and the size range over which the cluster-size distribution exhibits power-law scaling decreases, and the power-law exponent decreases to approximately 1.5 .

Spiral turbulence in a continuous excitable medium. We also consider a simple but realistic reaction-diffusion model proposed by Bär and Eiswirth [9] that describes the oxidation of $\mathrm{CO}$ on $\mathrm{Pt}(100)$. It is based on a piecewise linear version of the FitzHugh-Nagumo model [10] in which a delayed production of the inhibitor is introduced:

$$
\begin{gathered}
\partial_{t} u=-\frac{1}{\epsilon} u(u-1)\left(u-\frac{\mathrm{v}+b}{a}\right)+\Delta u, \\
\partial_{t} \mathrm{v}=f(u)-\mathrm{v} .
\end{gathered}
$$

The time scale relationship between the fast activator $u$ and the slow inhibitor $v$ is regulated by the parameter $\epsilon$, and the parameters $a$ and $b$ determine the excitation threshold. The production of the inhibitor is delayed according to the function $f(u)$, which results from fitting an experimentally obtained nullcline [11]:

$$
f(u)=\left\{\begin{array}{cc}
0 & u<1 / 3 \\
1-6.75 u(u-1)^{2} & 1 / 3 \leqslant u \leqslant 1 \\
1 & u>1 .
\end{array}\right.
$$

As a consequence of the specific form of $f(u)$, two unstable steady states appear in addition to the rest state, which play an essential role in the appearance of spiral turbulence in this model $[9,12]$. For a range of parameter values, a backfiring event (reexcitation in the wave back) gives rise to the breakup of a spiral wave. The resulting spiral turbulence, sustained by the collision of spiral arms and backfiring events, is shown in Fig. 2(a). Binary images of the patterns, with respect to a threshold value $u_{t h}$ that captures the essential features of the original spiral patterns, were generated for the coherent cluster analysis [see, for example, Fig. 2(b)]. The probability distribution of the coherent space-time clusters follows a power-law scaling to a good approximation over the parameter range of the spiral turbulence [see, Fig. 2(c)]. The power-law scaling is robust with respect to
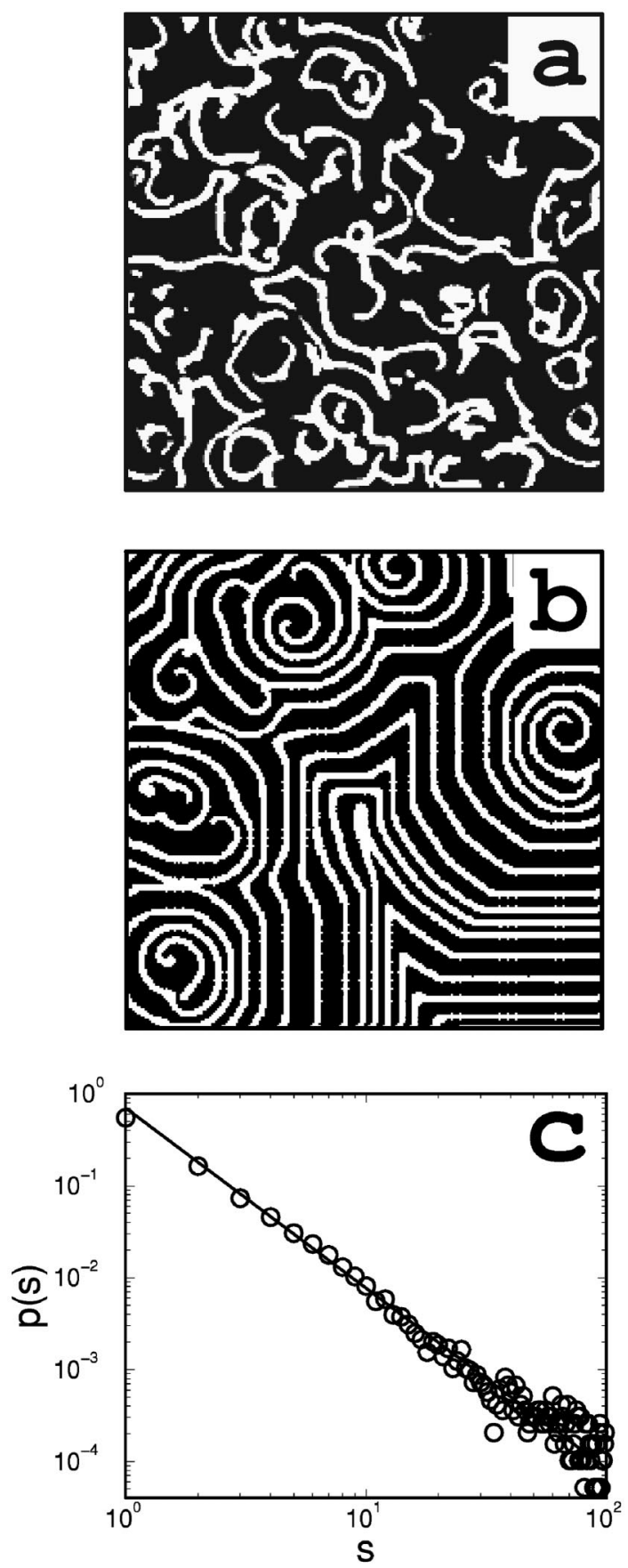

FIG. 1. Turbulent patterns obtained from the cellular automaton model, Eq. (1), with $\lambda=0.1, \gamma=10^{-3}, b=1.0$, and the coupling parameter $K=0.08$ (a) and 0.10 (b). The lattice spacing $a=1.0$, the time step $\Delta t=0.05$, and the refractory period was set to $4 \Delta t$. An asymmetric perturbation that yields a single spiral was chosen for the initial conditions. (c) Cluster-size distribution (10 000 clusters) for $K=0.08$ obtained from a series of 250 snapshots of an array of size $200 \times 200$. The scaling range can be approximated by the power law $p(s)=b s^{\alpha}$, with an exponent $\alpha \approx-1.9$.

changes in the threshold for values above $u_{t h}=0.7$, which represents the unstable focus of the system, although the actual slope (or scaling exponent) increases slightly with increasing $u_{t h}$, since a larger threshold value corresponds to a systematic decrease of cluster sizes. In contrast to the discrete model, Eq. (1), the scaling exponent does not depend significantly upon the bifurcation parameter $\epsilon$ (in the range 

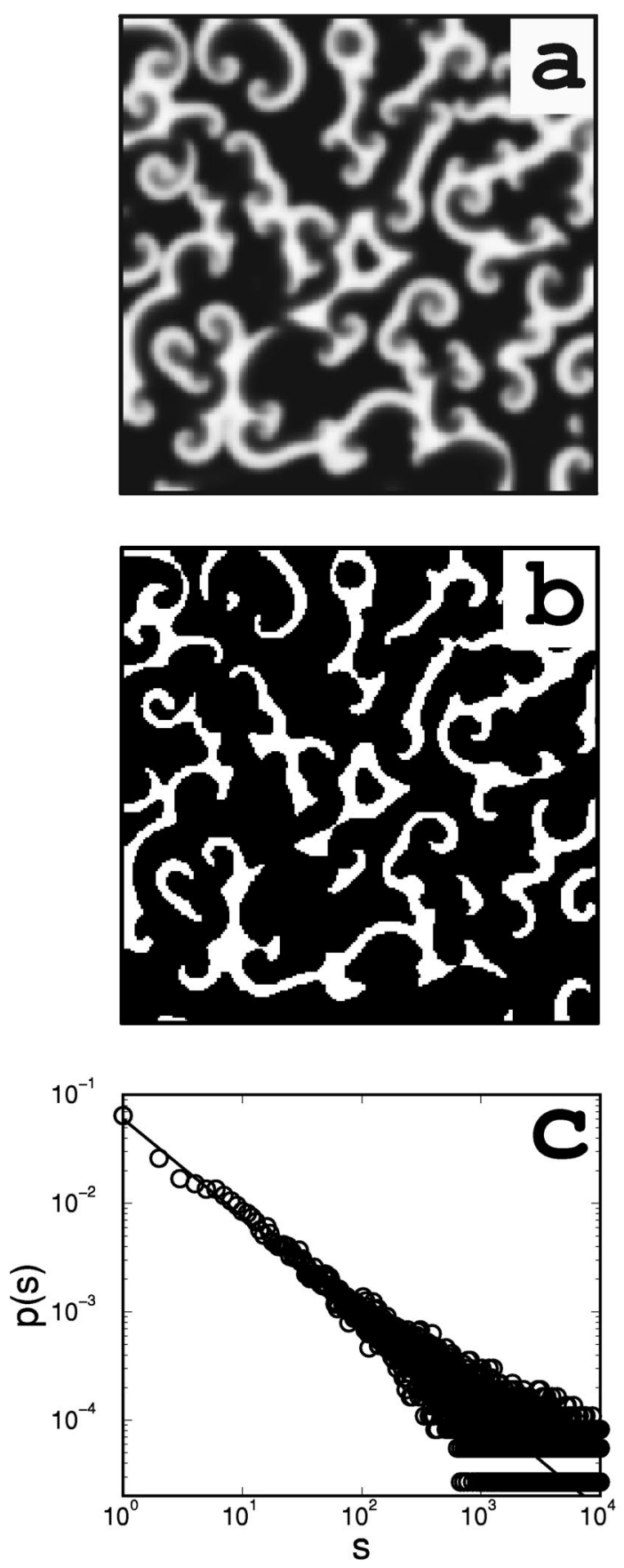

FIG. 2. (a) Typical spatiotemporal pattern of the surface model, Eq. (3), in the parameter regime of spiral turbulence. The equations were numerically integrated by an explicit Euler method (integration step $\delta t=0.014)$ using a nine-point approximation of the Laplacian on a domain of $300 \times 300$ grid points (where the unit grid size was 0.33), with the parameter values $a=0.84, b=0.07, \epsilon$ $=0.08$. An asymmetric perturbation that yields a single spiral was chosen for the initial conditions. (b) Corresponding binary image, where white regions designate an activator concentration of $u$ $>0.8$. (c) Probability distribution of coherent spatiotemporal clusters (37000 clusters), where the space-time cube for the analysis consists of approximately $10^{6}$ binary layers, and each layer [as in panel (b)] is taken every time step of $5 \delta t$. The scaling range can be approximated by the power law $p(s)=b s^{\alpha}$, with an exponent $\alpha \approx$ -1.0 . The power law is robust with respect to doubling the spatial size of the medium to $450 \times 450$ grid points.
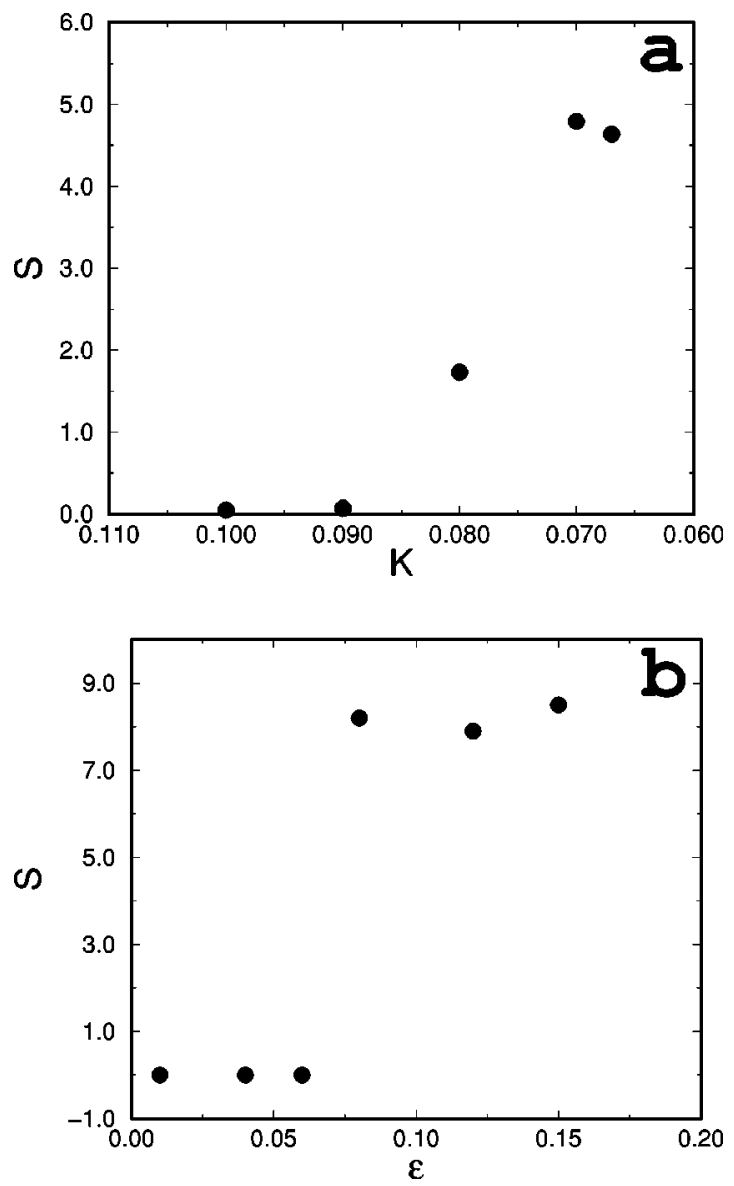

FIG. 3. Spatiotemporal entropy (a) as a function of the control parameter $K$ for the cellular automaton model, Eq. (1), and (b) as a function of $\epsilon$ for the reaction-diffusion model, Eq. (3). All other parameter values are the same as in Figs. 1 and 2.

$[0.08,0.15])$, although the rate of backfiring events increases with $\epsilon$. This indicates that the size distribution of coherent structures (or decomposed waves) is relatively independent of the backfiring rate.

Spatiotemporal entropy. The decomposition of the spatiotemporal patterns into coherent space-time clusters allows the behavior to be characterized in terms of notations borrowed from statistical physics. We first define the union of all clusters $n_{s}$ of spatiotemporal size $s$ to be in the $s$-cluster class $c_{s}$. Adding their sizes, one obtains the spatiotemporal volume $V_{s}$ of the class $c_{s}, V_{s}=s n_{s}$. The relative coverage $\mathrm{V}_{s}$ of the class $c_{s}$ is obtained by normalizing with respect to the total coverage of all classes $\mathrm{v}_{s}=s n_{s} / V_{\text {tot }}$, where $V_{\text {tot }}$ $=V_{1}+V_{2}+\cdots$. The relative coverage $\mathrm{v}_{s}$ can be expressed in terms of the normalized cluster-size distribution $p_{s}$ $=n_{s} / Z$, with the partition function $Z=\Sigma_{s} n_{s}$, i.e., $\mathrm{V}_{s}$ $=s p_{s} /\langle s\rangle$, where $\langle s\rangle$ is the first moment of the normalized cluster-size distribution. The relative coverage $\mathrm{V}_{s}$ has a simple and intuitive meaning: it gives the probability that any particular excited site belongs to the cluster class $c_{s}$.

The degree of homogeneity in this distribution of cluster classes reflects the disorder inherent in the spatiotemporal pattern and can be quantified by a spatiotemporal entropy,

$$
S=-\sum_{s} \mathrm{v}_{s} \ln \mathrm{v}_{s}=\ln \langle s\rangle-\frac{1}{\langle s\rangle} \sum_{s} s p_{s} \ln \left(s p_{s}\right) .
$$


The entropy vanishes if the excited sites belong to only one cluster class $c_{s}\left(\mathrm{v}_{s}=1\right)$, e.g., the spatiotemporal pattern consists of either a single cluster or clusters of only one size. A vanishing entropy does not necessarily require a "simple", initial state but can also arise from complex initial conditions, such as many spiral waves with randomly selected cores, if an inherent process for generating new cluster sources is lacking. The collision of uncorrelated clusters then occurs over a transient time to yield one surviving cluster. The artificial fracturing of waves at the boundaries does not affect the entropy, since the fractured waves belong to the same coherent parent structure. Applying the concept of spatiotemporal entropy to the patterns exhibited by the discrete and continuous models, we observe a marked increase in entropy at the transition from the ordered to the disordered state for both models, as shown in Fig. 3.

In the cellular automaton model, Eq. (1), the entropy increases smoothly with the degree of spatiotemporal disorder, reflecting an increasingly uniform probability distribution of cluster classes $c_{s}$. The low entropy values at higher values of $K$ reflect coherent spatiotemporal clusters arising from nucleation events mainly near defects (spiral cores and broken waves), while the higher values in the regime of spiral turbulence (at low values of $K$ ) correspond to nucleation events throughout the medium and a wide range of cluster sizes. In the reaction-diffusion model, Eq. (3), the entropy increases sharply at the bifurcation point marking the onset of backfiring and the transition from spiral patterns to the turbulent regime. The backfiring gives rise to a dramatic increase in the range of cluster sizes with a relatively homogeneous distribution of cluster classes, and, consequently, a sudden increase in the entropy.

In conclusion, we have suggested a method of quantifying and characterizing spatiotemporal turbulence that is based on the notation and concepts of statistical physics. The key feature of this description is the decomposition of spatiotemporal dynamics into space-time clusters of coherent wave activity. A realistic reaction-diffusion model with a backfiring instability as well as a cellular automaton model with nucleation dynamics exhibit power-law distributions of coherent cluster sizes, but with different scaling exponents. We have also introduced a spatiotemporal entropy, based on the size distribution of the space-time clusters, to quantify the degree of disorder. A sharp increase in the entropy is linked to the spontaneous generation of new waves, which collide with parent waves and thereby generate spatiotemporal disorder. A large entropy generally implies small predictability, i.e., it is difficult to predict the cluster class of an excited site in the turbulent regime. This notion of predictability, however, is different from the concept of Liapunov exponents for characterizing predictability in temporal chaotic systems. The coherent-cluster algorithm presented here allows an analysis of particular dynamical aspects of spiral turbulence, namely, the processes of wave nucleation and merging.

We thank the National Science Foundation (Grant No. CHE-9974336), the Office of Naval Research, and the Petroleum Research Fund for supporting this research.
[1] M.C. Cross and P.C. Hohenberg, Rev. Mod. Phys. 65, 851 (1993).

[2] M. Hildebrand, M. Bär, and M. Eiswirth, Phys. Rev. Lett. 75, 1503 (1995).

[3] S.M. Zoldi and H.S. Greenside, Phys. Rev. Lett. 78, 1687 (1997).

[4] S.M. Zoldi, J. Liu, K.M.S. Bajaj, H.S. Greenside, and G. Ahlers, Phys. Rev. E 58, R6903 (1998).

[5] P. Jung, Phys. Rev. Lett. 78, 1723 (1997).

[6] The notion of a coherent space-time cluster can be generalized such that a small cube is drawn around each active site, with a variable spatial side-length $d_{s}$ and a variable temporal side- length $d_{t}$, which allows one to analyze the patterns on adjustable scales. In this study, however, $d_{s}=d_{t}=1$ is kept fixed.

[7] R. FitzHugh, Biophys. J. 1, 445 (1961); J.S. Nagumo et al., Proc. IRE 50, 2061 (1962).

[8] Y. Hu, R.E. Ecke, and G. Ahlers, Phys. Rev. Lett. 74, 391 (1995).

[9] M. Bär and M. Eiswirth, Phys. Rev. E 48, R1635 (1993).

[10] D. Barkley, Physica D 49, 61 (1991).

[11] M. Bär, N. Gottschalk, M. Eiswirth, and G. Ertl, J. Chem. Phys. 100, 1202 (1994).

[12] M. Bär, M. Hildebrand, M. Eiswirth, M. Falcke, H. Engel, and M. Neufeld, Chaos 4, 499 (1994). 